\title{
Research review on cutting temperature and cutting vibration of titanium alloy
}

\author{
Yuqing $\mathrm{Li}^{1}$, Shuncai $\mathrm{Li}^{1}{ }^{1}{ }^{*}$ and, Qiu $\mathrm{Yu}^{2}$ \\ ${ }^{1}$ JSNU-SPBPU Institute of Engineering, Jiangsu Normal University, Xuzhou, China \\ ${ }^{2}$ School of Mechanical and Electrical Engineering, Jiangsu Normal University, Xuzhou, China
}

\begin{abstract}
The text should be set to 1 line spacing. The abstract should be centred across the page, indented $17 \mathrm{~mm}$ from the left and right page margins and justified. It should not normally exceed 200 words The titanium alloy called "strategic metal" is a typical refractory material that causes high temperature and vibration during cutting due to its high strength, low thermal conductivity and low elastic modulus. In the processing process, the tool wear is serious, and the accompanying cutting heat and cutting vibration are coupled to each other and change with time. Studying the temperature and vibration characteristics in the processing process is conducive to further understanding of cutting mechanism, better controlling temperature and vibration, improving cutting processing quality and reducing production cost. This paper reviews the current situation of cutting temperature and cutting vibration at home and abroad. Scholars focus on the influence of cutting factors on temperature and vibration, based on finite element simulation and mathematical modeling, and the research on temperature and vibration coupling of titanium alloy cutting should be further strengthened.
\end{abstract}

\section{Introduction}

Due to its high specific strength, excellent chemical corrosion resistance and biocompatibility, titanium alloy is widely used in the high-precision industries of human life, and is more applied in petroleum power generation, marine engineering and medical industries. As a not scarce resource on earth, titanium has not been widely used for many years, mainly due to the high application cost of titanium alloy.

With the rapid development and wide application of titanium alloys, the processing of titanium alloys is still a major industrial problem. Titanium alloy is a typical difficult-to-cut metal material. The main problems in the cutting process include: (1) small deformation coefficient, (2) high cutting temperature, (3) large cutting force per unit area, (4) the phenomenon of cold hardening is serious, (5) the tool is easy to wear. Therefore, it is of great significance to study thoroughly and systematically the turning temperature and turning vibration characteristics of titanium alloy during machining.

In recent years, many scholars at home and abroad have studied the temperature and vibration characteristics of titanium alloy turning from theories, experiments, finite element simulation, and predictive modeling.

\section{Current status of cutting temperature}

Due to its high strength and low thermal conductivity of titanium alloy, the contact area of the tool and the workpiece produces high pressure and temperature, while all the energy consumed is almost converted to thermal energy. Cutting heat is generally transmitted from chips, tools, work-pieces and surrounding media. The high temperature and strong vibration of the titanium alloy are cut due to its high strength, low thermal conductivity and low elastic modulus. Reasonable control of cutting temperature can reduce the impact of thermal deformation, reduce tool wear, extend the service life of tools, and thus stabilize the processing precision and processing quality.

\subsection{Factors affecting the cutting temperature}

Practice has proved that the workpiece material, tool material and structure, processing mode and cutting parameters setting are all important factors affecting the cutting temperature.

Davies [1] reviews several widely used cutting temperature measurements, Shows how the temperature can be monitored during material removal, in comparison with criteria critical for removal of measured materials, provide a reference to scholars in the field of work; Han [2] performed high speed cutting and milling tests on Ti$6 \mathrm{Al}-4 \mathrm{~V}$ titanium alloy material, studied and analyzed the trend of cutting temperature under dry cutting, air jet and nitrogen jet; Zhou [3] used low-temperature cooling technology. The results show that low-temperature cooling can significantly improve the stress and thermal stress of cutting tools; Liu et al [4-7] combined with ultrasonic vibration vehicle cutting to study the changes in amplitude, cutting velocity and displacement in different directions, analyze the influence of the two

\footnotetext{
*Corresponding author e-mail: zsclsc@263.net
} 
cutting methods on the cutting force and maximum temperature changes and material processing properties; Venkatesan [8] made an experimental study on laser assisted machining of Inconel 718 using turning process, and analyzed the effects of laser cutting parameters (i.e. cutting speed, feed rate and laser power) on cutting force and cutting temperature; Xavior [9] carried out precision turning of Ti-6Al-4V titanium alloy. Based on the experimental values, analysis of variance was carried out to understand the effects of cutting speed, feed rate, cutting depth and tool tip radius on cutting force, surface roughness and cutting tool temperature.

\subsection{Use finite element simulation to monitor and predict cutting temperature}

With the development of computer science and technology, finite element simulation becomes the main means of study heat and cutting temperature. He et al [1011] used finite element analysis (FEA) technology to model titanium alloy, the influence of the cutting parameters on the cutting temperature is analyzed, a regression model of cutting parameters and cutting temperature was established to predict titanium alloy cutting temperature; Veeranaath [12] studied the changes of workability, cutting stress and temperature of PCBN tool in dry processing Ti-6Al-4V by FEA technology; Tian[13] performed numerical simulation of the highspeed milling temperature field of titanium alloy TC4, the influence of the cutting parameters on the front and rear blade temperature and its distribution is studied; Based on the fluent solid energy equation, Du [14] constructed the finite element model of heat transfer on the tool chip contact surface in the second deformation zone, and discussed the effects of coating material, coating thickness and tool chip actual contact area on heat transfer when the coated tool was cutting H13 hardened die steel; Yan [15] combined with FEA technology to establish a 3 D cutting finite element model of TC4 titanium alloy with three elements, we analyze and compare the cutting force of three different coating tools; He [16] uses FEA technology to establish a cutting model, the effect of the cutting parameters on the cutting temperature field and the residual stress field is analyzed for simulation; combined with the Johnson-Cook Law of Materials, Zhang [17] established a three-dimensional finite element model (FEM) for oblique cutting and studied the distribution of cutting force, temperature and residual stress during oblique cutting of TC21 alloy; Wang [18] uses two finite element software under the same simulation parameters to analyze the cutting temperature distribution and the trend of cutting temperature with the cutting speed.

\subsection{Predictive modeling using mathematical methods}

In addition to the simulation analysis of cutting temperature using finite element software, many scholars also use mathematical models to establish analytical models related to cutting temperature, and predict the change of cutting temperature during the cutting process, with more high efficiency.Qu [19] establishes the mathematical model of D406A CNC cutting temperature and cutting force and determines the parameters in the model by cutting test; Ulutan [20] and Yan [21] predict the temperature during processing based on a thermal modeling technology which can greatly reduce the calculation time without causing much impact on the accuracy. Mohring [22] proposes a numerical iterative method for calculating the temperature of the contact area between the chip and the tool.The temperature of the tool measured on the front and rear knife faces is compared with the calculated temperature.Shan [23] developed an improved analytical model based on the mobile heat source method to predict the distribution of the cutting temperature in the orthogonal cutting of the titanium alloy TC4, showing that the relative difference in the predicted temperature was $0.49 \%$ to $9 \%$, indicating its high predictive power accuracy. Dayan [24] used the general full factor method to design the test, and established three prediction models of surface roughness, temperature and metal removal rate according to the obtained test data. The established model is in good agreement with the test results.Pervaiz [25] combines traditional finite element machining simulations with computational hydrodynamic models to establish a new method to analyze the temperature distribution of cutting tools to predict cutting heat and knife tip temperature during machining; Imbrogno [26] developed a new $3 \mathrm{D}$ finite element model to predict cutting force, temperature and corresponding microstructural changes in semi-refined Ti-6Al-4V under dry low temperature, and calibrated and verified.

\section{Current research status of cutting vibration}

Titanium alloy causes sharp cutting vibrations during cutting due to its lower elastic modulus and high strength. When the cutting vibration becomes large to a certain extent, the tool vibration directly affects the tool wear situation, and by monitoring the tool vibration can obtain information about the tool wear, improve the production quality and efficiency of the cutting [27].To this end, scholars study the influence of cutting parameters on the cutting vibration, the structure of the machine tool itself, the structure of knives and other titanium alloy cutting methods.

\subsection{Impact factors of cutting vibration}

Practice has proved that the workpiece material, tool material and structure, processing mode and cutting parameters setting are all important factors affecting the cutting temperature.

Sun [28] performs the first systematic study of the numerical and theoretical aspects of the high-speed vibrational cutting process of Ti-6Al-4V, help to deepen the understanding of the vibration effect of metal cutting, provide practical guidance for retraining and utilization of vibration assistance processing; Yan [29] studied the change of tool vibration with cutting parameters during the processing of TA2 pure titanium and TC4 titanium 
alloy; Zhou [30] goes through external circular vehicle machining of TA15 titanium alloy, the vibration signal characteristic values were collected and extracted, the influence law of the cutting amount on the cutting vibration and the surface roughness is analyzed, explain the effect of the cutting amount on the vibration; Zhu [31] systematically investigated the relationship between tool wear, chip morphology, and cutting vibration, tool wear was found to aggravate the cutting vibration, thus aggravating the chip edge wear.

\subsection{Monitor and predict tool wear based on cutting vibration}

Reasonable monitoring of cutting vibration can reduce the zigzag cutting chips caused by titanium alloy with high strength and low heat conductivity, and aggravate the vibration of the tool system, and then weaken the wear of the tool and the deterioration of surface processing quality.

Scholars analyze the optimal processing conditions of titanium alloy by building a prediction model. Silva [32] proposes a model to predict milling force, which is suitable for milling of ring tool and considers the cutting vibration effect of processing. Combined with FEM simulation analysis software, Patil [33] performs twodimensional FEM transient simulation and experimental characterization of titanium alloy under ultrasonic vibration assisted cutting condition using DEFORM software. Wang [34] uses the wavelet characteristics and support vector machine mode recognition algorithm to establish a milling vibration recognition system, and divide the vibration signal into three categories.

\subsection{Measurements of reducing cutting vibration}

The design of special structure knife rack, special shape tool and ultrasonic vibration auxiliary cutting methods can have a good effect on reducing cutting vibration. By manufacturing the hollow elements of special structure in the rack shaft, Vogel [35] greatly reduces the vibration amplitude of the tool and greatly improves the production quality of the piece. The texture tool proposed by Suzuki [36] can effectively cause process damping and inhibit vibration, which can play a good role in the experiments compared to ordinary tools. Rao [37] found that a new tool with a micro porous pattern on the front blade and side of the tool can reduce the friction of the front blade and thus reduce the vibration. Zheng [38] developed a rotating vibration control system based on multiple time delay, and numerical simulation found that the designed controller can effectively inhibit the cutting vibration and improve the stability of the cutting process. Identifying the dynamics of the cutting process based on working mode analysis, Kim [39] proposes a new vibration monitoring method that can determine the stability level of cutting before the vibration becomes unstable. Martin [40] studied the influence of cutting parameters on the vibration of machined parts. The experimental results show that the feed rate is the most important factor affecting the machining effect. Chen [41] proposes a novel nested artificial neural network that is more accurate to predict cutting states such as cutting vibration and surface roughness compared to conventional RSM and linear regression models. Compared to the traditional mathematical model in the new method, the newly proposed neural network class model has too high judgment coefficient and lacks the tests of the unfamiliar test set.

\section{Conclusion}

The temperature and vibration generated during the actual cutting process are coupled to each other and change over time. Based on the theoretical analysis, the scholars and scholars studied the temperature and vibration of titanium alloy cutting. However, the following points can be made to improve the cutting temperature and vibration.

(1) In terms of testing: At present, there are many research results on a single measurement method of cutting temperature and cutting vibration, and there are few research results on the simultaneous collection of cutting temperature and vibration. Therefore, in future research, a simultaneous measurement system for cutting temperature and vibration can be built, which provides an experimental basis for the analysis of the coupling characteristics between cutting temperature and vibration.

(2) Intelligent prediction: Most of the current research literature analyzes the influence of cutting parameters and tool structure parameters on cutting temperature and vibration through experimental testing and theoretical research, and uses a large number of numerical simulation methods such as finite element models to analyze temperature and vibration characteristics. Fewer scholars have established vibration and temperature prediction models, and more vibration and temperature prediction models can be established in the future to provide a reference solution for the current problem of the difficulty of measuring the turning temperature of titanium alloys in factories.

(3) In terms of coupling characteristics analysis: At present, most of the literature establishes the empirical formula of turning temperature on cutting parameters or the prediction model of cutting vibration on turning parameters. Few documents establish the fitting model between cutting vibration and temperature, and study the relationship between the two The coupling characteristics. Therefore, in the follow-up research, the research on the coupling characteristics between cutting temperature and turning vibration needs to be further strengthened.

\section{Acknowledgments}

This work was supported by the Postgraduate Research \& Practice Innovation Program of Jiangsu Province (KYCX21_2618), Joint scientific research project of Sino foreign cooperative education platform in Jiangsu Province, the Science and Technology Plan Project of Xuzhou City (KC20188), and Undergraduate Innovation Training Program (202010320033Z). 


\section{References}

1. J. Davim: Machining of Titanium Alloys (Springer Heidelberg New York Dordrecht London, England 2014)

2. M.L. Han, Y.M. Li and W. Zhao: Tool Engineering, Vol. 42 (2008) No.6, p.10.

3. B. Zhou, L. Hou, R. Tang and Q. Zhang: Combined machine tool and automatic machining technology, (2015) No.7, p.10.

4. A.J. Liu, Z.J. Zhou and B.L. Hu: Tool Engineering, (2017) No.12, p.40.

5. Z.T. Hu, N. Qin and F. Liu: Machinery Design \& Manufacture, (2018) No.2, p.164.

6. Y.F. Gao, H. Chen and Y. Jiang: Journal of Nanchang University (Engineering Edition), Vol. 39 (2017) No.3, p.263.

7. Y.S. Xu, P. Zou and W. Wang: Journal of Northeastern University (Natural Science Edition), Vol. 38 (2017) No.1, p.95.

8. K. Venkatesan, R. Ramanujam and P. Kuppan: Procedia Engineering, Vol. 97 (2014), p.1637.

9. M.A. Xavior and R. Vinayagamoorthy: Procedia Engineering, (2014) No.97, p.308.

10. Z.X. He, Y.H. Hu, C.J. Yin: Journal of Chinese Agricultural Mechanization, Vol. 35 (2014) No.4, p. 124 .

11. J.B. Sun, Y.H. Hu, L. He,: Machine Tool \& Hydraulics, Vol. 44 (2016) No.5, p.68.

12. V. Veeranaath: Materials Today: Proceedings, (2018) No.5, p.19547.

13. X.M. Tian, J.G. Ren and J.H. Zhou: Tool Engineering, Vol. 46 (2012) No.10, p.37.

14. J. Du, T.T. Zhou and J.J. Zhang, et al: Tool Engineering, (2018) No.5, p.51.

15. S. Yan, M.J. Wang, X.K. Hu: Combined machine tool and automatic machining technology, (2014) No.10, p.135.

16. X.J. He, G.D. Chen and T. Nelias: Mechanical Science and Technology for Aerospace Engineering, Vol. 30 (2011) No.12, p.2116.

17. Y.C. Zhang, T. Mabrouki and D. Shen, et al: International Journal of Material Forming, Vol. 4: (2011) No.1, p.11.

18. H.F. Wang, T. Xiao and W.G. Wu: Machinery Design \& Manufacture, (2012) No.09, p.48.

19. Z.X. Qu, and L.F.Zhang: NC [J]. Tool technology, Vol. 43: (2009) No.4, p.51-56.

20. D. Ulutan, I. Lazoglu and C. Dinc: Journal of Materials Processing Technology, Vol. 209 (2009) No.2, p.1111.

21. S.J.Yan, D.H.Zhu and K.J.Zhuang: Journal of Materials Processing Technology, Vol. 214: (2014) No.12, p.2985.

22. H.C. Möhring, C. Kushner and M. Storchak: CIRP Annals, Vol. 67: (2018) No.1, p.61.
23. C.W. Shan, X. Zhang and B. Shen, et al: Chinese Journal of Aeronautics, Vol. 32: (2019), p.759.

24. G.M. Dayan, R.H. Kishore, V. Praveen: Materials Today: Proceedings, Vol. 18: (2019), p.2191.

25. S. Pervaiz, I. Deiab and E.M. Wahba, et al: Procedia CIRP, Vol. (2014) No.17, p.750.

26. S Imbrogno, S Sartori, A Bordin, et al: Procedia CIRP, Vol. (2017) No.58, p. 475.

27. R. Kataoka and E. Shamoto: Precision Engineering, VoL. 55 (2019), p.322

28. S. Zongtao, S. Fei and M. Wei: International Journal of Mechanical Sciences, (2018) No.148, p.510

29. S.J. Yan, B.C. Xu and Q. Xin, et al: Manufacturing Technology \& Machine Tool, (2018) No.8, p.129

30. P.P. Zhou, J.Q. $\mathrm{Li}$ and L.F. Xu, et al: Tool Engineering, VoL. 50 (2016) No.5, p.21

31. Z.J. Zhu, J. Sun and J.F. Li, et al: The International Journal of Advanced Manufacturing Technology, VoL. 83 (2016) No.9-12, p.1477

32. V.A.D. Silva, R. Pederiva and H.K. Neto, et al: The International Journal of Advanced Manufacturing Technology, (2018) No.94, p.2115

33. S. Patil, S. Joshi and A. Tewari, et al: Ultrasonics, VoL. 54 (2013) No.2, p.694

34. Z.G. Wang, S. Nakashima, M. Larson: Procedia CIRP, (2014) No.17, p.523.

35. F. A.M. Vogel, S. Berger, E. Ozkaya, et al: Procedia Manufacturing, (2019) No.40, p.32.

36. N. Suzuki, W. Takahashi, H. Igeta, et al: CIRP Annals, VoL. 69 (2020), p.93.

37. C.M. Rao, S.S. Rao and M.A. Herbert: Journal of Manufacturing Processes, VoL. 36 (2018), p.93.

38. P.X. Zheng and X.H. Long: Theoretical and Applied Mechanics Letters, VoL. 3 (2013) No.6, p.e063003.

39. S. Kim and K. Ahmadi: Mechanical Systems and Signal Processing, VoL.130 (2019), p.315.

40. S. Martín-Béjar, F. Trujillo, C. Bermudo, et al: Procedia Manufacturing, VoL.41 (2019), p.835.

41. Y. Chen, R.L. Sun, Y. Gao, et al: Measurement, VoL.98 (2017), p.25. 